\title{
Predictive management of polarized light in liquid crystal devices based on average and flicker molecular tilt
}

\author{
A. Márquez, F. J. Martínez-Guardiola, J. Francés, S. \\ Gallego, I. Pascual, et al.
}

A. Márquez, F. J. Martínez-Guardiola, J. Francés, S. Gallego, I. Pascual, A. Beléndez, "Predictive management of polarized light in liquid crystal devices based on average and flicker molecular tilt," Proc. SPIE 11136, Optics and Photonics for Information Processing XIII, 1113609 (6 September 2019); doi: $10.1117 / 12.2530285$

SPIE Event: SPIE Optical Engineering + Applications, 2019, San Diego, California, United States 


\title{
Predictive management of polarized light in liquid crystal devices based on average and flicker molecular tilt
}

\author{
A. Márquez ${ }^{1,2,}$, F. J. Martínez-Guardiola ${ }^{1,2}$, J. Francés ${ }^{1,2}$, S. Gallego ${ }^{1,2}$, I. Pascual ${ }^{2,3}$, A. Beléndez ${ }^{1,2}$ \\ ${ }^{1}$ Dept. de Física, Ing. de Sistemas y T. Señal, Universidad de Alicante, P.O. Box 99, E-03080, Alicante, Spain \\ ${ }^{2}$ I.U. Física Aplicada a las Ciencias y las Tecnologías U. de Alicante, P.O. Box 99, E-03080, Alicante, Spain \\ ${ }^{3}$ Dept. de Óptica, Farmacología y Anatomía, Univ. de Alicante, P.O. Box 99, E-03080, Alicante, Spain
}

\begin{abstract}
We incorporate the prediction of flicker to a semiphysical and analytical model describing the angular and wavelength dependencies of retardance in parallel aligned liquid crystal (PA-LC) devices. This makes the model unique due to the wide range of calculation it offers while keeping its simplicity. Prediction of the modulation of retardance and its associated flicker relies on the fitting of the equivalent tilt angle of the molecules as a function of applied voltage. Specific results are given for liquid crystal on silicon (PA-LCoS) microdisplays, central to many spatial light modulation applications such as the generation of structured polarized beams. Experimental characterization results at arbitrary angles and wavelengths prove the predictive capability of the model. To highlight the richness of situations with PA-LCoS devices, we provide results for two different digital addressing sequences producing different levels of flicker. We focus on the application of the PA-LCoS as a polarization state generator (PSG) and we emphasize the ability of our approach to evaluate the performance across the visible spectrum and for a wide range of incidence angles. Our approach offers novel capabilities in the generation of arbitrary states of polarization, both fully and partially polarized.
\end{abstract}

Keywords: Polarization, Liquid crystal on silicon displays, Polarimetry, Spatial light modulation, Flicker, Displays.

\section{INTRODUCTION}

Parallel-aligned liquid crystal on silicon (PA-LCoS) microdisplays have found widespread application in many spatial light modulation applications ${ }^{[1]-[3]}$, which typically require phase-only modulation. Within this context, in recent years, a wide interest has arisen dealing with the generation and detection of unconventional polarization structured wavefronts ${ }^{[4][5]}$ in which PA-LCoS devices play a central role ${ }^{[6][7]}$. The device can be easily modeled as a variable linear retarder, however, the existence of flicker in many of these devices has resulted in the proposal of more complete models and characterization methods, such as in the time-averaged Stokes polarimetric technique ${ }^{[8]}$.

Recently, as an alternative to "black-box" models, we proposed and demonstrated a semiphysical model for PA-LC devices $^{[9]}$, where only the most relevant internal parameters remained. This semiphysical model provides the voltage dependent retardance for a very wide range of incidence angles (from 0 to $45^{\circ}$ ) and any wavelength in the visible spectrum. We apply a straightforward reverse-engineering approach to fit its three parameters from a very limited amount of experimental measurements. This is the most simplified model still showing predictive capability for PA-LCoS devices. Recently, we further demonstrated that the fitted values obtained for two of the three parameters in the model are equal to the actual values. Thus, the model shows not only predictive capability but it also enables to probe into the internal properties of PA-LCoS devices ${ }^{[10][11]}$. A very significant progress was demonstrated recently ${ }^{[12]}$, where we showed that the model predicts not only the retardance but also the magnitude of its flicker at other wavelengths and incidence angles different from the measured ones. This enables to calculate the degradation effects due to phase flicker and if they are limiting the performance of specific applications. Furthermore, from the point of view of the physics in the device, it is very appealing connecting the flicker in the retardance with instabilities in the tilt angle for the LC director. In this work we show the model and demonstrates its wide range of potential application.

Optics and Photonics for Information Processing XIII, edited by Khan M. Iftekharuddin,

$1113609 \cdot$ @ 2019 SPIE · CCC code: 0277-786X/19/\$21 · doi: 10.1117/12.2530285 


\section{MODELLING RETARDANCE AND FLICKER BASED ON MOLECULAR TILT}

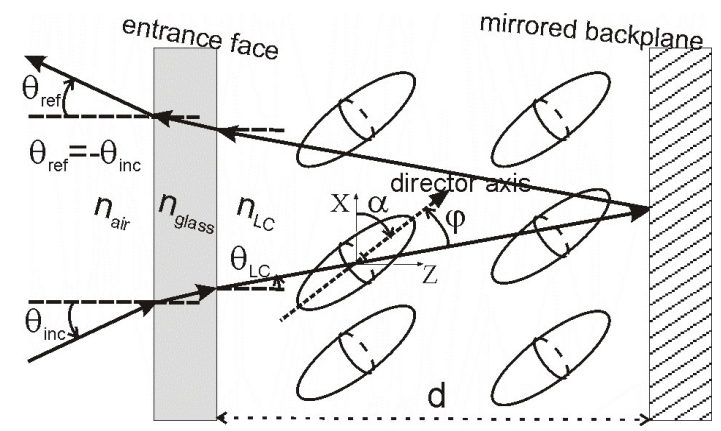

Figure 1. Diagram for the PA-LC cell considered in the semiphysical model.

Next, we present the semiphysical model for PA-LC devices, whose agreement with experimental measurements was already verified for a commercial reflective PA-LCoS device ${ }^{[9]}$. We have further shown ${ }^{[10]}$ that the fitted values obtained for two of the three parameters in the model are equal to the actual values, thus these parameters are physically meaningful. In Figure 1 we show the general diagram considered for a reflective cell with a cell gap $d$. Incidence plane and LC director are along the XZ plane. LC molecules have their director axis (optical axis) aligned at an angle $\phi$ with respect to the traversing light beam direction. $\theta_{L C}$ is the refraction angle in the LC medium. The director axis tilts an angle $\alpha$ with respect to the entrance face as a function of the applied voltage $\mathrm{V}$. This is the only voltage dependent magnitude, i.e. $\alpha(V)$ . At the backplane the light beam is reflected and a second passage is produced across the LC layer whose effect is equivalent to a forward propagation at an angle $-\theta_{i n c}$. In the model we define two off-state parameters, combination of the LC indices ordinary and extraordinary, no and ne, together with the cell gap $d$. They are $O P L=d n_{o}$ and $O P D=d \triangle n$, which correspond respectively to the magnitudes of the optical path length (OPL) for the ordinary component and the optical path difference (OPD) between extraordinary and ordinary components. Proper derivation leads to the following analytical expression for retardance ${ }^{[9]}$,

$$
\Gamma=\frac{2 \pi}{\lambda} \frac{O P L}{\cos \theta_{L C}}\left[\frac{1+(O P D / O P L)}{1+(O P D / O P L) \cos ^{2} \phi}-1\right]
$$

According to Figure 1, angle $\phi$ is given by,

$$
\phi\left(\theta_{i n c}, V\right)=\frac{\pi}{2}+\alpha(V) \mp \theta_{L C}\left(\theta_{i n c}\right)
$$

, where the minus (plus) sign applies for the forward (backward) passage. The total retardance in the PA-LCoS is given by the addition of the forward and backward retardances. In the case of normal incidence and LC director axis parallel to the entrance face, then Eq. (1) simplifies into the well-known expression $\Gamma=2 \pi d \Delta n / \lambda$.

We note that in our previous works ${ }^{[9][10]}$, as candidate to simplified physical model, we also evaluated the exact expressions for a homogeneous uniaxial anisotropic plate ${ }^{[13][14]}$. Both models disregard the fact that the tilt angle of the LC director changes across the cell. Our model adds a second approximation, since it does not take into account the double refraction. However, we obtained that both simplified models show the same level of predictive capability, with the benefit that our model produces a much simpler expression and reduces the number of parameters when compared with these exact expressions. Additionally, when fitting the parameters, our model converges to a unique solution, whose values are equal to the real physical values in two of its three parameters: OPD and tilt angle $\alpha(V)$. This was not the case with the exact expressions.

The reverse-engineering approach to obtain the values for the parameters in our semiphysical model employs the average retardance $\bar{\Gamma}$ measurements provided by the time-averaged Stokes polarimetric technique ${ }^{[8]}$. Off-state measurements, 
LCoS switched-off, are used to obtain OPL and OPD using standard non-linear iterative fitting procedure. Once these two parameters are fixed, then we proceed with the on-state measurements to obtain the tilt angle $\alpha(V)$ for each applied

voltage. The time-averaged Stokes polarimetric technique provides both the average retardance $\bar{\Gamma}$ and its flicker amplitude $\Delta \Gamma$ at each applied voltage ${ }^{[9]}$. According to this, we define the maximum and the minimum expected retardance value respectively as $\Gamma_{+}=\bar{\Gamma}+\Delta \Gamma$ and $\Gamma_{-}=\bar{\Gamma}-\Delta \Gamma$. In this paper, using these values in the fitting procedure we obtain the tilt angles $\alpha_{+}(V)$ and $\alpha_{-}(V)$ associated respectively with the experimental values $\Gamma_{+}$and $\Gamma_{-}$. Then, the flicker in the tilt angle at each applied voltage value $\Delta \alpha(V)$ is calculated as the average absolute value, i.e. $\Delta \alpha=\left|\alpha_{+}-\alpha_{-}\right| / 2$. In the next Section, we will show the results obtained.

\section{SIMULATED RESULTS}

In the experiments we use a commercially available PA-LCoS microdisplay, model PLUTO distributed by the company HOLOEYE. Its backplane is digitally addressed ${ }^{[15]}$ and different pulse width modulation (PWM) addressing schemes (digital addressing sequences) can be generated by the driver electronics ${ }^{[15][16]}$. We have selected two electrical sequences exhibiting a clearly different scale of fluctuations, whose configuration files are provided with the software. They correspond to the configurations labeled as "18-6 633 2pi linear" and "5-5 633 2pi linear".

The time-averaged Stokes polarimetric measurements have been obtained with a Stokes polarimeter, model PAX5710VIS$\mathrm{T}$ distributed by the company THORLABS ${ }^{[8]}$. Measurements have been taken at various angles of incidence $\left(3^{\circ}, 23^{\circ}, 35^{\circ}\right.$ and $\left.45^{\circ}\right)$ and for three wavelengths $(473,532$ and $633 \mathrm{~nm})$ sampling the visible spectrum. We will use the measurements taken at $3^{\circ}$ and $35^{\circ}$ for calibration, and then the measurements at $23^{\circ}$ and $45^{\circ}$ will be used to analyze the predictive capability of the semiphysical model. The figure of merit to be minimized combines two squared differences: on one hand between theoretical and experimental retardance values normalized by the experimental value, and on the other hand between the theoretical and experimental ratios of the retardance values at $3^{\circ}$ and $35^{\circ}$ incidence normalized by the experimental ratio. These two normalized squared differences are added up for the three wavelengths.

Table 1. Fitted OPD and OPL obtained using the experimental retardance values in Table 1.

\begin{tabular}{cccc}
\hline$\lambda(\mathrm{nm})$ & 633 & 532 & 473 \\
\hline $\mathrm{OPD}(\mu \mathrm{m})$ & 0.5275 & 0.5937 & 0.6489 \\
$\mathrm{OPL}(\mu \mathrm{m})$ & 2.5761 & 2.6064 & 6.8925 \\
\hline
\end{tabular}

First, we obtain the values for OPL and OPD using the off-state retardance measurements at angles of incidence $3^{\circ}$ and $35^{\circ}$. In Table 1 we show the fitted values. We fix these values in the theoretical expressions for the semiphysical model. Then, we use the on-state average retardance and flicker amplitude values for incidence angles at $3^{\circ}$ and $35^{\circ}$ as the experimental values for the fitting procedure with the theoretical expressions. From the average retardance fit we obtain the tilt angle as a function of voltage $\alpha(V)$. We proceed identically with the maximum $\Gamma_{+}=\bar{\Gamma}+\Delta \Gamma$ and the minimum $\Gamma_{-}=\bar{\Gamma}-\Delta \Gamma$ experimental values to calculate, as explained in Section 2, the flicker in the tilt angle $\Delta \alpha(V)$. In Fig. 2(a) we show the tilt angle $\alpha(V)$ as a function of the gray level addressed with the graphics card, which is related with the applied voltage. We see the resulting values for the two sequence format configurations, 5-5 and 18-6, previously presented. The tilt angle increases monotonically with the gray level, i.e. the applied voltage increases with the gray level.

The result obtained for the tilt flicker $\Delta \alpha(V)$ for the two sequences is shown in Fig. 2(b) as a function of gray level. Both sequences exhibit a very different behavior for the tilt flicker: for sequence 5-5 is clearly smaller and increases with gray level, whereas for 18-6 it reaches a maximum value about $4.5^{\circ}$ and then stays basically constant. We also note in the tilt flicker a series of jumps, which are related with one of the bit planes in the digital sequence changing its state from $\mathrm{ON}$ to OFF or viceversa ${ }^{[15][16]}$. 

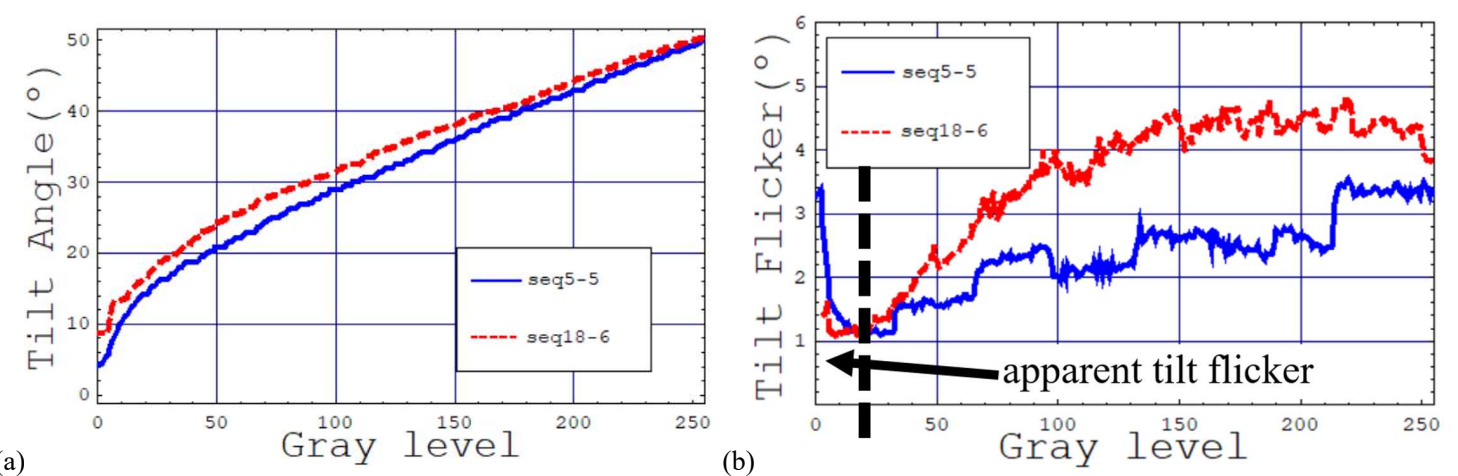

Fig. 2. Tilt angle (a) and tilt flicker (b) for sequence formats 5-5 and 18-6 as a function of gray level.

At very low gray levels (low tilt angles) we see that the tilt flicker increases, especially for sequence 5-5 where it reaches a value larger than $3^{\circ}$. This flicker is not real: we showed in an earlier paper ${ }^{[17]}$ (see Fig. 6 therein) that at 0 gray level there is no flicker for these sequences 5-5 and 18-6. The origin for this "apparent" tilt flicker comes from the kind of experimental measurements that we use to fit the parameters in the model, as we recently analyzed ${ }^{[12]}$. In Fig.2(b), we have marked with a vertical dashed line the point where the tilt flicker reaches a minimum: at about 20 gray level ( $14^{\circ}$ tilt angle). At lower gray levels (tilt angles), the real tilt flicker actually continues decreasing (in the off-state this would be exactly $0^{\circ}$ ). Thus, in the plot we have labelled this region as "apparent tilt flicker".
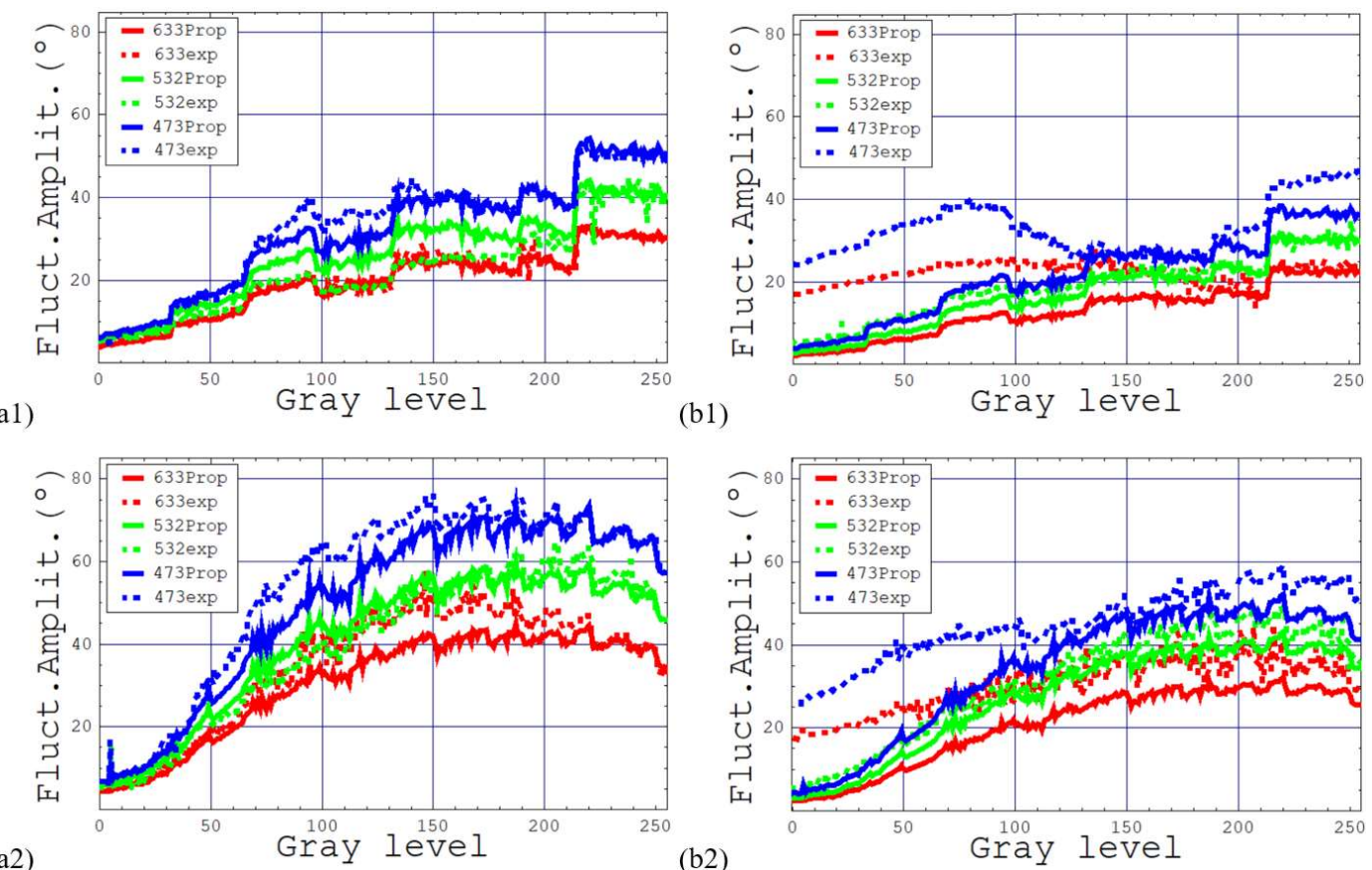

(b1)

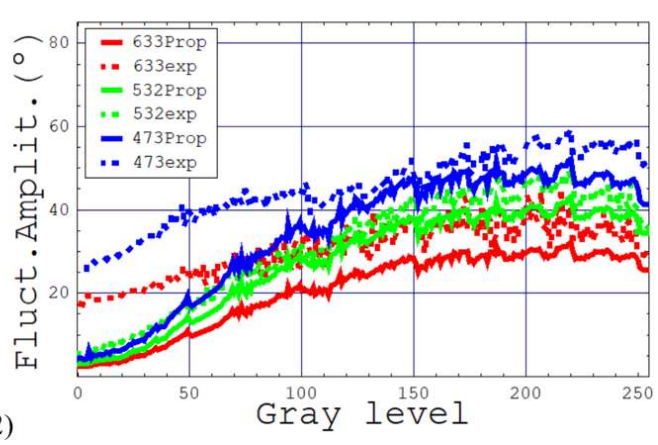

Fig. 3. Experiment (dots) and theoretical prediction with the proposed model (line) for sequence formats 5-5 ((a1) and (b1)) and 18-6 ((a2) and (b2)), for the wavelengths 473, 532 and $633 \mathrm{~nm}$ and for incidence at $23^{\circ}((\mathrm{a} 1)$ and (a2)), and at 45 ((b1) and (b2)).

Now, we analyze the usefulness of the model and its calculated average and flicker retardance values to predict the SOP and DoP for the light reflected by the PA-LCoS device. We consider that the incident SOP corresponds to right-handed circular light. We provide measurements and calculations for the wavelength $473 \mathrm{~nm}$, for the sequence $5-5$ and for different angles of incidence. In Fig. 4 we show the maximum range of SOP modulation that is possible with our current PA-LCoS and for sequence 5-5. When using the shorter wavelength, $473 \mathrm{~nm}$, and with light incident perpendicularly to the entrance face of the PA-LCoS is when we obtain the larger modulation range. Agreement between experiment and model is very good, thus showing the accuracy and usefulness of the model in order to design appropriate working configurations 
(incidence angles, illumination wavelenths), gray level ranges,... adapted to the specific application in mind. We note that more results were given recently ${ }^{[12]}$.

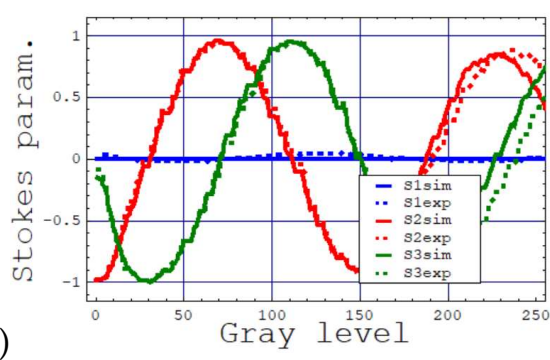

(a)

. For sequence format 5-5 and for incident SOP right-handed circular, for $473 \mathrm{~nm}$ and at $3^{\circ}$ incidence, in plots (a) for the Stokes parameters and in (b) for DoP, experiment (dots) and theoretical prediction with the proposed model (line), and in plots (c) representation on the Poincaré sphere, where the dark line is simulation and gray line is experiment.

\section{CONCLUSIONS}

We have demonstrated a complete model for parallel aligned liquid crystal (PA-LC) devices for both retardance and flicker. It is the first time that flicker in the retardance can be predicted for PA-LC devices. The model provides the equivalent flicker in the tilt angle of the LC molecules. The model is valid across the whole visible spectrum and for a wide range of incidence angles. We demonstrate its usefulness to generate both fully and partially polarized states of polarization

\section{ACKNOWLEDGEMENTS}

Work funded by Ministerio de Economía, Industria y Competitividad (Spain), projects FIS2017-82919-R (MINECO/AEI/FEDER, UE) and FIS2015-66570-P (MINECO/FEDER)); by Generalitat Valenciana (Spain), project PROMETEO II/2015/015 and GV/2019/021; and Universidad de Alicante (Spain), project GRE17-06.

\section{REFERENCES}

[1] Z. Zhang, Z. You, and D. Chu, "Fundamentals of phase-only liquid crystal on silicon (LCOS) devices," Light Sci. Appl. 3, 1-10 (2014).

[2] G. Lazarev, P.-J. Chen, J. Strauss, N. Fontaine, A. Forbes, "Beyond the display: Phase-only liquid crystal on Silicon devices and their applications in photonics," Opt. Express 27, 16206-16249 (2019).

[3] A. Márquez, A. Lizana, "Special Issue on Liquid Crystal on Silicon Devices: Modeling and Advanced Spatial Light Modulation Applications," Appl. Sci. 9, 3049 (2019).

[4] T. G. Brown, Q. Zhan, "Focus Issue: Unconventional Polarization States of Light," Opt. Express 18, 10775-10776 (2010).

[5] I. Moreno, J. A. Davis, K. Badham, M. M. Sánchez-López, J. E. Holland, D. M. Cottrell, "Vector Beam Polarization State Spectrum Analyzer", Sci. Rep. 7, 2216 (2017).

[6] X. Zheng, A. Lizana, A. Peinado, C. Ramirez, J. L. Martinez, A. Marquez, I. Moreno, and J. Campos, "Compact LCOSSLM based polarization pattern beam generator," J. Lightwave Technol. 33, 2047-2055 (2015).

[7] A. Cofré, A. Vargas, F. A. Torres-Ruiz, J. Campos, A. Lizana, M. M. Sánchez-López, and I. Moreno, "Dual polarization split lenses," Optics Express 25, 23773-23783 (2017).

[8] F. J. Martínez, A. Márquez, S. Gallego, J. Francés, I. Pascual, and A. Beléndez, "Retardance and flicker modeling and characterization of electro-optic linear retarders by averaged Stokes polarimetry," Opt. Lett. 39, 1011-1014 (2014).

[9] F. J. Martínez, A. Márquez, S. Gallego, J. Francés, I. Pascual, and A. Beléndez, "Effective angular and wavelength modeling of parallel aligned liquid crystal devices," Opt. Lasers Eng. 74, 114-121 (2015). 
[10] A. Márquez, J. Francés, F. J. Martínez, S. Gallego, M. L. Álvarez, E. M. Calzado, I. Pascual, and A. Beléndez, "Computational split-field finite-difference time-domain evaluation of simplified tilt-angle models for parallel-aligned liquid-crystal devices," Opt. Eng. 57, 037110 (2018).

[11] J. Francés, A. Márquez, F. J. Martínez-Guardiola, S. Bleda, S. Gallego, C. Neipp, I. Pascual, and A. Beléndez, "Simplified physical modeling of parallel-aligned liquid crystal devices at highly non-linear tilt angle profiles," Opt. Express 26, 12723-12741 (2018).

[12] A. Márquez, F. J. Martínez-Guardiola, J. Francés, S. Gallego, I. Pascual, A. Beléndez, "Combining average molecular tilt and flicker for management of depolarized light in parallel-aligned liquid crystal devices for broadband and wide-angle illumination," Opt. Express 27, 5238-5252 (2019).

[13]P. Yeh, C. Gu, [Optics of Liquid Crystal Displays], John Wiley \& Sons (1999).

[14] A. Lien, "Extended Jones matrix representation for the twisted nematic liquid-crystal display at oblique incidence", Appl. Phys. Lett.57, 2767-2769 (1990).

[15]F. J. Martínez, A. Márquez, S. Gallego, M. Ortuño, J. Francés, A. Beléndez, and I. Pascual, "Electrical dependencies of optical modulation capabilities in digitally addressed parallel aligned LCoS devices," Opt. Eng. 53, 067104 (2014).

[16] A. Hermerschmidt, S. Osten, S. Krüger, T. Blümel, "Wave front generation using a phase-only modulating liquidcrystal based micro-display with HDTV resolution," Proc. SPIE 6584, 65840E (2007).

[17]F. J. Martínez, A. Márquez, S. Gallego, J. Francés, and I. Pascual, "Extended linear polarimeter to measure retardance and flicker: application to liquid crystal on silicon devices in two working geometries," Opt. Eng. 53, 014105 (2014). 